University of Nebraska - Lincoln

DigitalCommons@University of Nebraska - Lincoln

CSE Conference and Workshop Papers

Computer Science and Engineering, Department of

2017

\title{
Wireless Underground Channel Diversity Reception With Multiple Antennas for Internet of Underground Things
}

Abdul Salam

University of Nebraska-Lincoln, asalam@cse.unl.edu

Mehmet C. Vuran

University of Nebraska-Lincoln, mcvuran@cse.unl.edu

Follow this and additional works at: http://digitalcommons.unl.edu/cseconfwork

Part of the Computer Engineering Commons, Electrical and Computer Engineering Commons, and the Other Computer Sciences Commons

Salam, Abdul and Vuran, Mehmet C., "Wireless Underground Channel Diversity Reception With Multiple Antennas for Internet of Underground Things" (2017). CSE Conference and Workshop Papers. 303.

http://digitalcommons.unl.edu/cseconfwork/303

This Article is brought to you for free and open access by the Computer Science and Engineering, Department of at DigitalCommons@University of Nebraska - Lincoln. It has been accepted for inclusion in CSE Conference and Workshop Papers by an authorized administrator of

DigitalCommons@University of Nebraska - Lincoln. 


\title{
Wireless Underground Channel Diversity Reception With Multiple Antennas for Internet of Underground Things
}

\author{
Abdul Salam and Mehmet C. Vuran \\ Cyber-Physical Networking Laboratory \\ Department of Computer Science \& Engineering \\ University of Nebraska-Lincoln, Lincoln, NE 68588 \\ Email: \{asalam, mcvuran\}@cse.unl.edu
}

\begin{abstract}
Internet of underground things (IOUT) is an emerging paradigm which consists of sensors and communication devices, partly or completely buried underground for real-time soil sensing and monitoring. In this paper, the performance of different modulation schemes in IOUT communications is studied through simulations and experiments. The spatial modularity of direct, lateral, and reflected components of the UG channel is exploited by using multiple antennas. First, it has been shown that bit error rates of $10^{-3}$ can be achieved with normalized delay spreads $\left(\tau_{d}\right)$ lower than 0.05 . Evaluations are conducted through the first software-defined radio-based field experiments for UG channel. Moreover, equalization has a significant impact on the performance improvement of an IOUT system. An 8-Tap DFE (decision-feedback equalizer) adaptive equalizer achieves better performance. It is also found that DBPSK, and DPSK are more suitable for digital communications in the UG channel without adaptive equalization. Then, two novel UG receiver designs, namely, 3W-Rake and Lateral-Direct-Reflected (LDR) are developed and analyzed for performance improvement. It has been shown that with a three antenna LDR design, BER of lower than $10^{-5}$ can be achieved. The BER of these two approaches are compared and the LDR has been shown to perform better.
\end{abstract}

\section{INTRODUCTION}

The realization of the high data rate, and long-range wireless underground (UG) communications is one of the major enabling factors of the Internet of Underground Things (IOUT) [13]. The delay spread of the UG channel causes performance degradation and leads to frequency selective fading [16]. This effect restricts the data rates in the UG channel and results in irreducible bit error rates (BER). The impact of the delay spread, soil moisture, soil type, and frequency selective fading due to the delay spread is an important issue in the UG communications channel [13], [16]. The UG communications system should have the ability to adjust to soil dynamics such as soil moisture variations, and also support high data rate communications with low BER [6]. Due to these factors, characterization and performance analysis of the UG channel is a challenging task.

In [6], we have employed the channel capacity as a tool for IOUT system performance analysis. However, to date, a study to analyze the performance of digital modulation schemes in an IOUT system employing the wireless UG channel as a communication medium is unavailable. These effects are investigated in this work by using the detailed impulse response data of the wireless underground communications channel [16].

In this paper, we develop a model to generate the channel impulse response from measured data and use it to simulate a fully functional IOUT communications system using conventional modulation schemes, i.e., pulse-amplitude modulation (PAM), differential phase shift keying (DPSK), quadrature phase shift keying (QPSK), m-ary quadrature amplitude modulation (MQAM), and Gaussian minimum-shift keying (GMSK). The purpose of this work is to analyze the impact of the normalized RMS delay spread on the digital modulation in the UG channel by using measured UG channel responses, and to optimize the IOUT communications system design parameters such as modulation scheme and bit error rates. Adaptive equalization of the UG frequency selective fading channel has also been considered in this work and it has been shown that the use of adaptive equalization in the UG channel leads to performance improvements.

Moreover, in this paper, issue of design of a UG receiver based on lateral, direct and reflected components of the wireless UG channel is addressed. We develop two novel techniques, 1) a single antenna $3 \mathrm{~W}$-Rake receiver to combat multipaths effects, 2) a spatial diversity multi-antenna LateralDirect-Reflected (LDR) receiver, which exploits the spatial modularity and angular diversity found in the propagation environment of the wireless UG channel. We describe the $3 \mathrm{~W}-$ Rake and LDR system models, and analyze their performance in different soil types, depths, distances, and soil moisture levels. The results reported in this work are useful for design and optimization of a wireless IOUT communications system.

The rest of the paper is organized as follows: The related work is discussed in Section II. The description of wireless UG channel model is given in Section III. System models are described in Section IV. Performance evaluations are performed in Section V. We conclude in Section VI. 


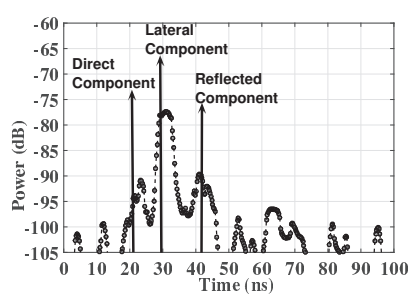

(a)

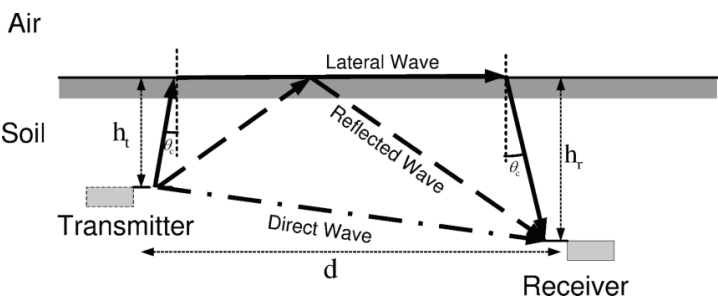

(b)

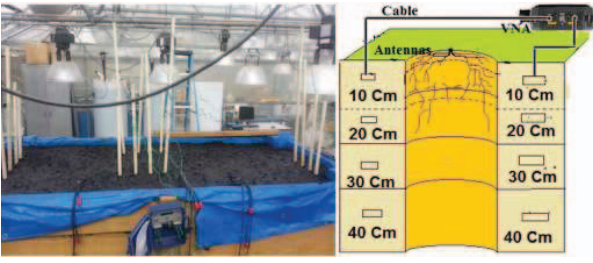

(c)

Fig. 1: (a) An example power delay profile (PDP) of the impulse response model of the wireless UG channel [16], (b) L, D and R-Wave in the UG Channel [7], (c) The indoor testbed [16].

\section{RELATED WORK}

Underground communications in IOUT has many applications in precision agriculture [2], [4], [8], [17], [14], [15], [21], [22], border monitoring [3], [19], land slide monitoring, and pipeline monitoring [18], [23]. A detailed characterization of the wireless UG channel has been provided in [16]. Impacts of soil type and moisture on the capacity of multi-carrier modulations are discussed in [13]. However, to the best of our knowledge, no performance analysis of digital modulation schemes has been carried out in the electromagnetic (EM) based UG wireless communication channel. Capacity analysis [11], has been done for magneto-inductive (MI) based UG communications [1], [20], but it cannot be readily applied to IOUT because the spatial multipath modularity does not exist in MI, and sender-receiver coils have to be parallel to each other in MI-communications.

To the best of our knowledge, this is the first work to analyze the performance of digital wireless UG channel receivers in IOUT and to analyze the impact of normalized delay spread, and different modulation schemes on the bit error rate of wireless UG channel.

\section{BACKGROUND}

Despite the recent developments in wireless UG communications, the communication ranges are still limited for many potential applications. Therefore, advanced communication techniques, designed based on the unique characteristics of the wireless UG channel, are required. A robust IOUT communication system can be designed through physical insight into the propagation characteristics of the wireless UG channel. A channel model for UG communications has been developed in [16] and has been validated empirically. Direct, lateral, and reflected components have been identified at the UG receiver. An example of the power delay profile (PDP) of wireless UG channel has been shown in Fig. 1(a). EM based communications in the UG channel are carried out through three different paths (Fig. 1(b)). Direct wave (D-wave) propagates through the soil in the line-of-sight (LOS). Reflected wave (R-wave) is reflected from the soil air interface and reaches at the receiver. The lateral wave ( $\mathrm{L}$-wave) propagates along the soil-air interface and continuously diffuses inward to reach at the receiver. L-wave is the strongest component as it suffers low attenuation when passes through the air along the soil-air interface as compared to the reflected and direct wave which undergoes higher attenuation due to the high losses in soil medium.

The UG channel impulse response is expressed as a sum of direct, reflected and lateral waves [16]:

$h_{u g}(t)=\sum_{l=0}^{L-1} \alpha_{l} \delta\left(t-\tau_{l}\right)+\sum_{d=0}^{D-1} \alpha_{d} \delta\left(t-\tau_{d}\right)+\sum_{r=0}^{R-1} \alpha_{r} \delta\left(t-\tau_{r}\right)$,

where $\mathrm{L}, \mathrm{D}$, and R are number of multipaths; $\alpha_{l}, \alpha_{d}$, and $\alpha_{r}$ are complex gains; and $\tau_{l}, \tau_{d}$, and $\tau_{r}$ are delays associated with lateral wave, direct wave, and reflected wave, respectively.

In [16], measurements have been taken both in indoor testbed and field settings. The indoor testbed and experiment layout has been shown in Fig. 1(c). In the indoor testbed, three sets of four dipole antennas are buried at a distances of $50 \mathrm{~cm}$, at the depths of $10 \mathrm{~cm}, 20 \mathrm{~cm}, 30 \mathrm{~cm}$, and $40 \mathrm{~cm}$. Silt loam and sandy soils are used in the indoor testbed. In the outdoor testbed, antennas are buried at $20 \mathrm{~cm}$ depth up to $12 \mathrm{~m}$ distance. Agilent FieldFox N9923A Vector Network Analyzer (VNA) is used to measure channel transfer functions. More details about the testbed development, measurement procedures, experiments, and results can be found in [16].

\section{System Models}

Let $u(t)$ be the baseband input to the UG channel, the convolution of the $h_{u g}$ with $u(t)$ gives the received signal output waveform.

$$
z(t)=u(t) * h_{u g},
$$

which can be expressed as:

$$
z(t)=\sum_{l=0}^{L-1} \alpha_{l} u\left(t-\tau_{l}\right)+\sum_{d=0}^{D-1} \alpha_{d} u\left(t-\tau_{d}\right)+\sum_{r=0}^{R-1} \alpha_{r} u\left(t-\tau_{r}\right) .
$$

In this analysis, we normalize UG channel delay spread $\tau_{d}$ based on the sample period $T$ and RMS delay spread $\left(\tau_{r m s}\right)$, where $\tau_{d}$ is given as:

$$
\tau_{d}=\frac{\tau_{r m s}}{T} .
$$

Bandwidth can be expressed as $B=1 / T$. For the modulation schemes considered, signaling waveform $u(t)$ is convolved with $h_{u g}$. Both rectangular, and raised cosine pulses are used for signaling. Raised cosine filter helps to minimize ISI and is realized through raised cosine spectrum with roll-off factor $\beta$. At the receiver, we compute the BER performance. 
The UG channel impulse responses, $h_{u g}$, used in this analysis are sampled from measured power delay profiles (PDP) in different soils under different soil moisture conditions at different depths and distances. In this work, we do not use coding schemes, and results reported in this work are without employing coding. A detailed analysis of the error correcting coding schemes in the UG channel is given in [5]. Use of coding improves the performance of the system at the cost of increased complexity and energy consumption of the UG receiver. Performance analysis using conventional receiver approach results in high error rates (Section V). A 3W-Rake receiver design is developed next to mitigate the effects of multipath fading and to improve system performance.

\section{A. UG 3W-Rake Receiver}

In this section, a UG receiver design without spatial diversity is presented. Since three components, namely, direct, lateral, and reflected wave are resolvable, this approach is based on the use of RAKE [12] to resolve three independently faded components by exploiting the high diversity in the three components. UG $3 \mathrm{~W}$-Rake consists of three branches, one for each of the lateral, direct, and reflected components. In the UG $3 \mathrm{~W}-$ Rake receiver, each branch correlates the received signal with its specified component to separate the three components.

Due to the UG multi-path fading phenomena, the received instantaneous signal-to-noise ratio (SNR) is a random process. Therefore, we average the Additive White Gaussian Noise (AWGN) error probability over the probability density function (pdf) of the SNR $\gamma_{b}$. The average BER probability, $P_{b}\left(\bar{\gamma}_{b}\right)$, of the UG $3 \mathrm{~W}-$ Rake is calculated as [12]:

$$
P_{b}(\bar{\gamma})=\int_{0}^{\infty} P_{e \mid \gamma_{b}} p\left(\gamma_{b}\right) d \gamma_{b},
$$

where $\bar{\gamma}_{b}$ denotes the average SNR per bit, $P_{e \mid \gamma_{b}}$ is the conditional AWGN error probability, and $p\left(\gamma_{b}\right)$ is the pdf of SNR. Since, no close form solution of the pdf of the $\gamma_{b}$ is available for the UG channel, we determine $p\left(\gamma_{b}\right)$ from experimental UG channel impulse response measurements [16] by averaging $P_{e \mid \gamma_{b}}$ over the instantaneous SNR for each measured response. Since UG 3W-Rake can process multipaths in all the three components, received per bit SNR $\gamma_{b}$ is expresses as:

$$
\gamma_{b}=\sum_{l=0}^{L-1} \gamma_{l}+\sum_{d=0}^{D-1} \gamma_{d}+\sum_{r=0}^{R-1} \gamma_{r},
$$

where L, D, and R are number of multipaths; $\gamma_{l}, \gamma_{d}$, and $\gamma_{r}$ are gains associated with lateral wave, direct wave, and reflected wave, respectively. (6) can be rewritten as:

$$
\gamma_{b}=\frac{E_{b}}{N_{0}}\left[\sum_{l=0}^{L-1}\left|\gamma_{l}\right|^{2}+\sum_{d=0}^{D-1}\left|\gamma_{d}\right|^{2}+\sum_{r=0}^{R-1}\left|\gamma_{r}\right|^{2}\right],
$$

where energy per bit to noise PSD (power spectral density ratio), $\frac{E_{b}}{N_{0}}$, is given as:

$$
\frac{E_{b}}{N_{0}}=\frac{P_{t} T}{N_{0} P L},
$$

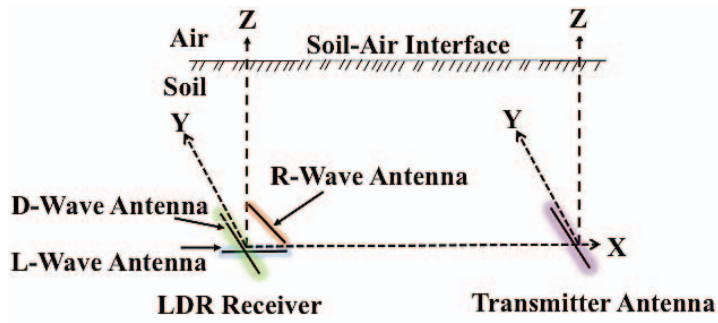

Fig. 2: LDR antenna orientation.

where $P_{t}$ is the transmitted power, $T$ is the sample period, $N_{0}$ is noise density, and $P L$ is the path loss.

Through this procedure, a discrete $p(\gamma)$ is approximated. Once $p(\gamma)$ is determined for $3 \mathrm{~W}$-Rake, the average bit error probability, $P_{b}(\bar{\gamma})$, is calculated by using (5). In the next section, we extend the idea of $3 \mathrm{~W}$-Rake to the Lateral-DirectReflected (LDR) case to exploit spatial modularity of the UG channel.

\section{B. LDR Receiver Design}

In this section, a novel LDR diversity reception technique is developed. LDR is based on the knowledge of the angular arrival, delay spreads, and travel paths of three EM wave components in the UG channel. This approach offers considerable performance improvement over the conventional matched filter based UG receiver and $3 \mathrm{~W}$-Rake. Three antennas are used to combine direct, lateral, and reflected wave multipath components, which eliminate multipath fading of the wireless UG channel. Delay spreads of the wireless UG channel presented in [16], provide a detailed insight into the propagation characteristics of the wireless UG channel. As discussed in Section III, there exists a natural spatial modulation (SM) in the UG channel in the form of direct, lateral, and reflected waves. However these three waves cause inter-symbol-interference and lead to performance deterioration of an IOUT system. By the LDR receiver design, these issues are addressed and performance is improved by eliminating interference between these three components.

LDR Antenna Orientation: We consider an IOUT system where both transmitter and receiver are buried underground. Transmitter has a single antenna, whereas, receiver has three antennas, each for one of the three components. For UG channel diversity reception, the following antennas are configured: the antenna designated to receive the $\mathrm{D}$-wave is at $90^{\circ}$ from the $\mathrm{x}$-axis; the $\mathrm{R}$-wave antenna is at a line connecting $x-z$ axis, with center at $45^{\circ}$ from $\mathrm{x}$-axis; whereas, $\mathrm{L}$-wave antenna is placed at $0^{\circ}$ from the $\mathrm{x}$-axis. For this orientation, to avoid any variations in receiver's axis, transmitter and receiver are assumed to be on the same depth on a straight line along the $\mathrm{x}$-axis. LDR antenna orientation is shown in Fig. 2.

LDR System Model: Based on the LDR antenna orientation of one transmitter antenna and three L, D, and R-wave antennas, the received signal is expressed as:

$$
\boldsymbol{z}=\boldsymbol{h}_{\boldsymbol{u g}} u+\boldsymbol{n}
$$

where $u$ is the transmitter's data symbol, $\boldsymbol{z}$ is a $3 \times 1$ received output vector, $h_{u g}$ is the channel vector representing the $\mathrm{L}$, 


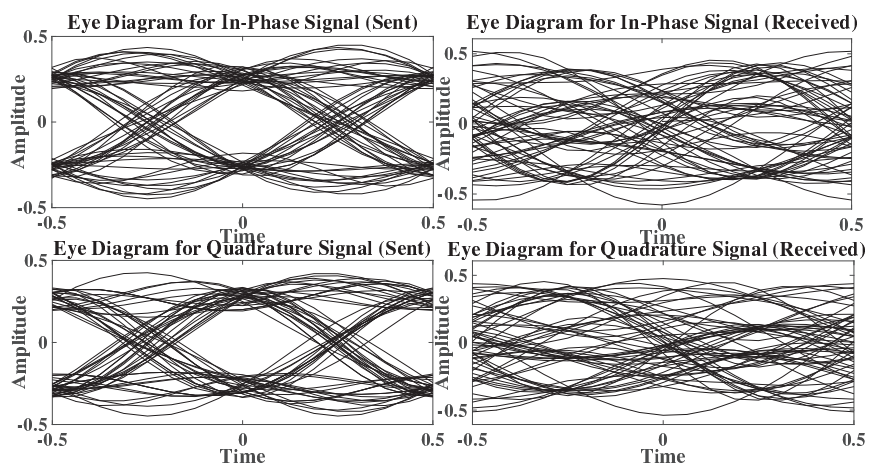

Fig. 3: QPSK eye patterns of transmitted and received signals.

$\mathrm{D}$, and R-wave channel response, and $\boldsymbol{n}$ is the $3 \times 1$ noise vector. For each component antenna, the channel response is separable and is denoted as $h_{d}, h_{l}$, and $h_{r}$, for the direct, lateral and reflected components, respectively. At the each receive component, the instantaneous SNR is defined as:

$$
\gamma_{i}=\frac{E_{b}\left|h_{i}\right|^{2}}{N_{0}}
$$

where $i$ represents the $L, D$, and $R$ components.

Optimum Maximum Ratio Combining (MRC-LDR): By using maximum ratio combining (MRC) [12], LDR can achieve three times SNR enhancement as compared to the SNR of a single antenna matched filter UG receiver:

$$
\gamma=\sum_{i=1}^{3} w_{i} \frac{E_{b}\left|h_{i}\right|^{2}}{N_{0}}
$$

where $w_{i}$ is the combining weight. MRC-LDR achieves the maximum gain, however the interference from the reflected components is still present. Therefore, to suppresses undesired interference adaptive switching and selection is presented next.

Adaptive Combining (AC-LDR): Based on the proximity of the LDR receiver, either the D-wave or L-Wave component is dominant at the receiver. AC-LDR exploits this by adaptively switching and selecting the strongest L, or D-Wave (RWave is not considered because it is the weakest component and results in performance degradation), such that:

$$
\gamma= \begin{cases}\frac{E_{b}\left|h_{L}\right|^{2}}{N_{0}}, & \text { if }\left|h_{L}\right|^{2}>\left|h_{D}\right|^{2}, \\ \frac{E_{b}\left|h_{D}\right|^{2}}{N_{0}}, & \text { otherwise. }\end{cases}
$$

The main difference between MRC-LDR and AC-LDR is that AC-LDR removes all the interference at the cost of channel gain. The average BER probability, $P_{b}\left(\bar{\gamma}_{b}\right)$, of the both LDR approaches is calculated as [12]:

$$
P_{b}(\bar{\gamma})=\int_{0}^{\infty} P_{e \mid \gamma_{b}} p\left(\gamma_{b}\right) d \gamma_{b},
$$

\section{Performance AnAlysis}

Since UG channel multipath power delay profile depends mainly on the soil type and moisture, depth, and distance of the UG transmitter and receiver, in this study, we simulate the UG channel with $\tau_{d}$ range of 0.4-0.002. In Section V-A, we

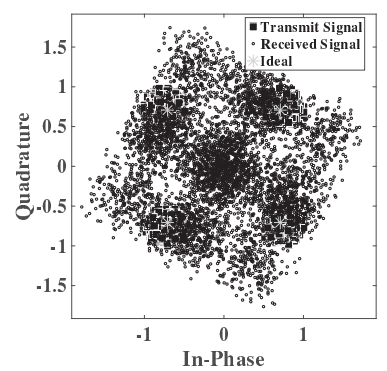

(a)

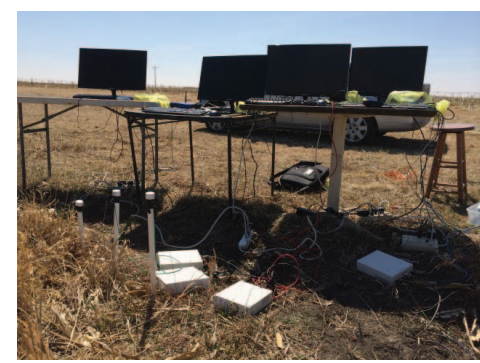

(b)
Fig. 4: a) Constellation diagram of QPSK, b) BER experiment layout in the silty clay loam soil.

analyze the performance of the coherent modulation schemes. Empirical evaluation results are presented in Section V-B. Differential detection schemes in UG channel are evaluated in Section V-D. Performance analyses of 3W-Rake and LDR are presented in Section V-E, and V-F, respectively. Finally, implementation issues are discussed in Section V-G.

\section{A. Coherent Detection}

Four digital modulation schemes, namely, PSK, QAM, PAM, and MSK are evaluated in this section. Performance of these four modulation schemes has been compared for an UG channel in silty clay loam soil. The soil moisture level is $0 \mathrm{CB}^{1}$ and $\tau_{r m s}$ is $25 \mathrm{~ns}$. The transmitter and receiver distance is $50 \mathrm{~cm}$ and these are buried at $20 \mathrm{~cm}$ depth. Our analysis reveals high error rates of higher than $10^{-1}$ for all four modulation schemes. In the UG channel propagation environment is highly degraded due to the multipath fading which is the main cause of the worst performance of the coherent modulation in the UG channel. Moreover, coherent modulation requires exact knowledge of the channel state. Due to higher delay spreads in the UG channel, reference symbol tracking is difficult to implement. It is also interesting to note that for $\tau_{d}$ range $0.002-0.4$, error rate does not change, which indicates that error floor is irreducible and does not depend on the sample time. This suggests that the performance of the digital modulations in the UG channel is severely effected by the multipath fading, and increasing the transmit power of the UG the transmitter will not result in reduction of error rate.

To further investigate the cause of high error rates, we plot the constellation and eye diagrams. In Fig. 3 and 4(a), the constellation and eye diagrams are shown for QPSK modulation in the UG channel. It can be observed from Fig. 3 that eye suffers from severe performance deterioration (both horizontal and vertical closure) due to inter symbol interference and large delay spreads between the three components. Complete eye closure due to these phenomena has led to high error rates. These simulations results are validated with empirical evaluations. Empirical results are shown in the next section.

\footnotetext{
${ }^{1}$ Soil moisture expressed as soil matric potential (CB); greater matric potential values indicate lower soil moisture and zero matric potential represents near saturation condition
} 


\section{B. Experimental Evaluation}

In this section, first, we describe the experimental setup and validation, and then results are shown.

1) Setup: To analyze the BER performance of the UG channel, we conducted experiments using GNU Radio [10] and Ettus N210 USRPs [9]. Dipole antennas in these experiments are buried at $20 \mathrm{~cm}$ depth at a distance of $50 \mathrm{~cm}$ in silty clay loam soil. Soil moisture level is $50 \mathrm{CB}$ and $\tau_{r m s}=25.67$ ns [16]. Transmitter-Receiver (TR) are synchronized by using a MIMO cable. Transmit power is $10 \mathrm{dBm}$. The operation frequency range is from $100 \mathrm{MHz}$ to $300 \mathrm{MHz}$. Normalized delay spread $\tau_{d}$ range is $0.005-0.43$. A series of sequences of 1000 bits are sent from transmitter using amplitude-shift keying (ASK) modulation. At the receiver side, error statistics of the channel are obtained by comparing the output with input. For each $\tau_{d}$, we calculate the bit error rate by adding the bits in error of each correct symbol and then dividing this sum by total number of bits in all symbols at receiver. Experimental setup is shown in Fig. 4(b).

2) Empirical Results: Evaluations are conducted through the first software-defined-radio (SDR) based field experiments for UG channel. BER results of empirical ASK are evaluated for $\tau_{d}$ range of 0.005-0.43. Empirical results also exhibit very high error rate (higher than $10^{-1}$ ) and show vulnerability of UG communications to the multipath fading of the UG channel. Since, transmitter and receiver are synchronized, and reference signal is available at the receiver, which confirms that, in UG channel, in addition to the timing and phase recovery issues which effect the performance of the coherent modulation schemes, an additional factor of delay distortion of three major multipath components significantly impacts the performance of coherent modulation techniques in the IOUT environment. In over-the-air (OTA) channels, use of adaptive equalization [12] is very effective against this type of performance deterioration (ISI and multipath fading). Therefore, we investigate the use of adaptive equalization to overcome these effects in the UG channel. In the next section, we analyze the performance of equalization in the UG channel.

\section{Performance of Equalization in the UG Channel}

In this section, we analyze of the performance of PSK modulation in the UG communication channel. PSK is used because adaptive equalization works best for constant modulus modulation [12] as compared to ASK. Three equalization scenarios are considered. All three uses training sequences for equalization. These three cases are explained below:

Case 1 - Single Tap Linear Equalizer: Single tap least-mean square (LMS) equalizer is used with phase and gain control. Modulation scheme is QPSK, and 50 blocks are transmitted in each simulation run. Case 2 - Eight Tap Linear Equalizer: In this case, simulations are performed by using eight tap linear recursive least square (RLS) equalizer with QPSK modulation and 50 blocks are transmitted. Case 3 - Adaptive Equalization: In this case, adaptive equalization is performed in the receiver using a decision-feedback equalizer (DFE) with two tap feedback weights and a six tap feedforward filters. The

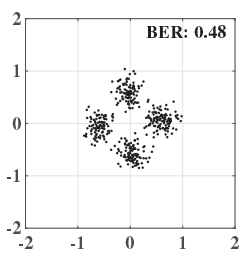

(a)

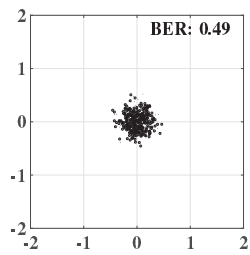

(d)

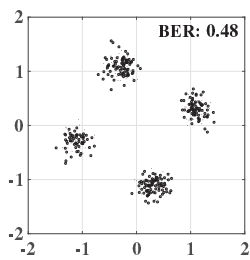

(g)

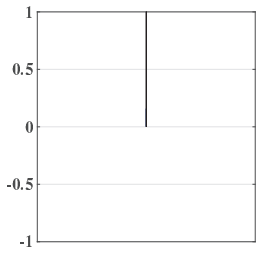

(b)

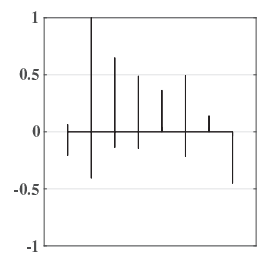

(e)

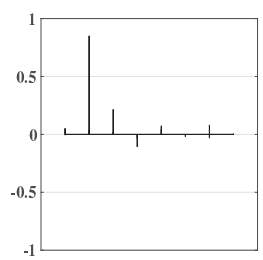

(h)

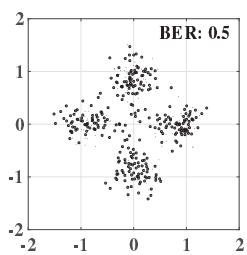

(c)

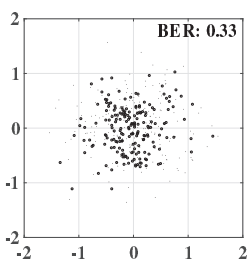

(f)

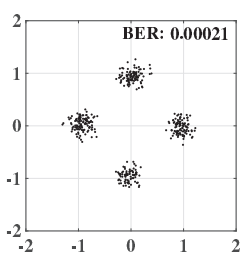

(i)
Fig. 5: Single tap linear equalizer: a) Received constellations b) Equalizer weights, c) Equalized constellations. Eights tap linear equalizer: d) Received constellations. e) Equalizer weights f) Equalized constellations. DFE (decision-feedback equalizer) with two tap feedback weights and a six tap feedforward filters: g) Received constellations, h) Equalizer weights, i) Equalized constellations.

DFE uses an eight-tap linear recursive least squares (RLS) equalizer with symbol spaced taps.

In Figs. 5(a)-5(c), results of the single tap linear equalizer are shown. Received constellation with equalization is shown in the Fig. 5(a) with BER of 0.48. Equalizer weights are shown in Fig. 5(b). It can be observed from Fig. 5(c) that use of single tap equalizer does not improve the receiver performance and BER remains unchanged (0.5). In Figs. 5(d)$5(\mathrm{f})$, performance of the eight tap linear equalizer is shown. In Fig. 5(d), the received constellation with BER of 0.49 is shown. It can be observed that increase in number of taps (Fig. 5(e)) has made some improvements, but received constellation was severely corrupted therefore it only results in minor gain and BER has reduced from 0.49 to 0.33 (Fig. 5(f)).

Adaptive equalization performance is shown in the In Figs. 5(g)- 5(i). Received constellation (Fig. 5(g)) has the BER of 0.48 , and it can be observed that use of 8-tap adaptive equalizer (Fig. 5(h)) has removed most channel distortions and results in BER less than $10^{-3}$. Improvements in the equalized constellations are clearly visible in Fig. 5(i). From these results, it can be observed that performance of an unequalized UG communication system is limited due to the UG channel propagation characteristics and inter symbol interference (ISI). Therefore, increase in the transmit power does not lead to substantial performance improvements. Hence, use 


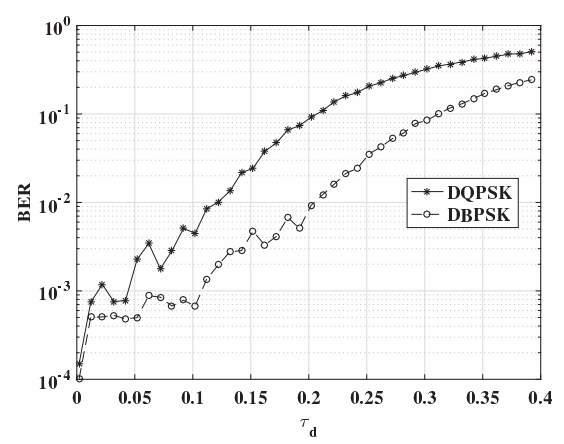

(a)

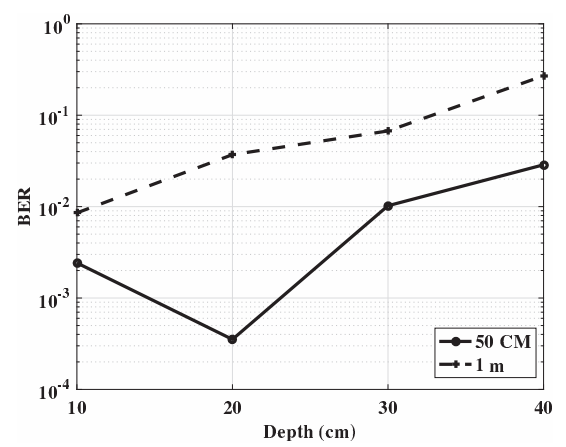

(b)

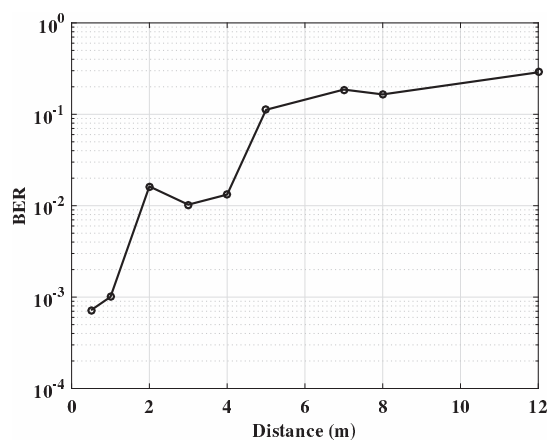

(c)

Fig. 6: Average BER performance comparison: (a) Differential BPSK and QPSK at $\tau_{r m s} 25 \mathrm{~ns}$, (b) With burial depth at $50 \mathrm{~cm}$ and $1 \mathrm{~m}$ distance in silt loam soil, (c) With distance at $20 \mathrm{~cm}$ depth for distances up to $12 \mathrm{~m}$ in silty clay loam soil.

of equalizer is required in UG communications for a reliable communication system design. By equalization, ISI is removed which leads to performance improvement. Our analysis shows that minimum size of DFE equalizer should be 8-tap with two tap feedback weights and a six tap feedforward filters. In the next section, we evaluate the performance of the differential detection schemes in the UG channel.

\section{Differential Detection}

In this section, performance of the UG channel communications is evaluated by using differential binary phase shift keying (DBPSK), and differential quadrature phase shift keying (DQPSK). In Fig. 6(a), BER performance comparison of DBPSK, and DQPSK for $\tau_{r m s} 25 \mathrm{~ns}$ is shown. Since soil moisture is a slowly changing phenomena, variations in the UG channel response are slow. The channel estimation (carrier accusation and tracking) is not required in the differential detection at the UG receiver. Instead, symbols received in the previous symbol period are used as phase reference in the current symbol period, therefore differential technique works better in the UG channel as compared to coherently detected modulation schemes. It can be observed that for normalized delay spread $\tau_{d}$ values of less than 0.1 error rate has decreased to $10^{-3}$ as compared to the $10^{-1}$ error rate of the coherent modulation schemes. It can also be observed that performance of the UG channel starts to degrade with higher $\tau_{d}$. For $\tau_{d}$ greater than 0.2 , error rate is higher than the $10^{-2}$ for both DBPSK and DQPSK. However, differential schemes still perform better than the $10^{-1}$ BER of coherent modulation.

\section{E. $3 W$-Rake Performance in UG Channel}

In this section, we evaluate the performance of UG $3 \mathrm{~W}$ Rake receiver. In the UG channel, SNR required for the target BER threshold is analyzed for different modulation schemes. Different factors such as soil type and soil moisture affects the UG communications. Therefore, we consider different representative scenarios of the $\mathrm{UG}$ communications in silt loam, sandy, and silty clay loam soils; for soil moisture level of 0-50 CB, at depths of $10 \mathrm{~cm}, 20 \mathrm{~cm}, 30 \mathrm{~cm}$, and $40 \mathrm{~cm}$; and distances up to $12 \mathrm{~m}$. As discussed in Section V-E, we need to determine the $p\left(\gamma_{b}\right)$ to compute the average BER, $P_{b}(\bar{\gamma})$, in the UG channel. We get the SNR from empirical impulse responses [13], [16] and evaluate performance for $\tau_{d}$ of 0.01 , and measured noise density of $1 E-15$, in PAM modulation.

In Fig. 6(b), average BER with burial depth at $50 \mathrm{~cm}$ and $1 \mathrm{~m}$ distance in silt loam soil is shown. BER for $40 \mathrm{~cm}$ depth are highest as compared to shallow depths. It can be observed that at $50 \mathrm{~cm}$ distance, BER first decreases from $10 \mathrm{~cm}$ to $20 \mathrm{~cm}$ depth, and then increases at $30 \mathrm{~cm}$ and $40 \mathrm{~cm}$ depth. This happens because at shallow $10 \mathrm{~cm}$ depth, reflections from surroundings affect the received signal. With increase in burial depth at $1 \mathrm{~m}$ distance, BER increases, which is caused by the additional attenuation of the EM waves at higher depths. Error rates further increase with increase in transmitter-receiver (TR) distance from $50 \mathrm{~cm}$ to $1 \mathrm{~m}$. BER in silty clay loam soil at $20 \mathrm{~cm}$ depth for distances up to $12 \mathrm{~m}$ is shown in Fig. 6(c). BER of $10^{-3}$ are observed for distances less than $1 \mathrm{~m}$, and BER of $10^{-2}$ can be achieved for distances up to $4 \mathrm{~m}$. For distances higher than $5 \mathrm{~m}$, error rates are higher than $10^{-1}$. Increase in propagation loss of the all three components with distance causes higher attenuation and lead to higher BER. Degradation in system performance can be improved by utilizing the error correcting codes [5] for larger distances in the UG channel.

In Fig. 7(a), change in average BER with soil moisture at $50 \mathrm{~cm}$ and $1 \mathrm{~m}$ distance in silt loam soil is shown. It can be observed that decrease in soil moisture from $10 \mathrm{CB}$ to $50 \mathrm{CB}$ leads to variations in BER for both $50 \mathrm{~cm}$ and $1 \mathrm{~m}$ distance. At $50 \mathrm{~cm}$ depth, from $10 \mathrm{CB}$ to $50 \mathrm{CB}$ change in soil moisture, BER decreased first and then increases as soil moisture decreases. This is caused by water repellency of soil texture where water infiltration is slowed momentarily at high soil moisture levels. Moreover, change in soil moisture impacts the attenuation through which UG channel undergoes due to the absorption of the EM waves by the water contained in the different horizons of the soil. In the next section, we evaluate the performance of the LDR technique.

\section{F. LDR Performance Analysis}

Let us now consider performance improvement with LDR. We use normalized delay spread, $\tau_{d}<0.1$. Results of the comparison of $3 \mathrm{~W}$-Rake with MRC-LDR and AC-LDR are shown in Fig. 7(b). It can be observed that both LDR outperform the $3 \mathrm{~W}$-Rake and substantial BER performance 

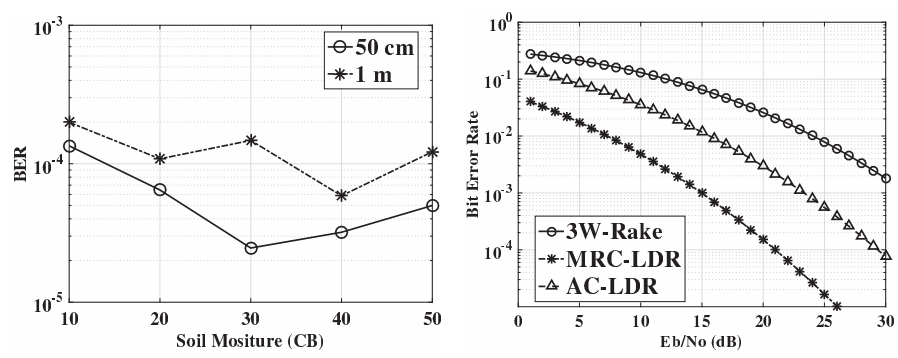

Fig. 7: a) Variations in average BER with change in soil moisture at $50 \mathrm{~cm}$ and $1 \mathrm{~m}$ distance in silt loam soil, b) Comparison of $3 \mathrm{~W}$-Rake, MRC-LDR, and AC-LDR.

improvement is realized for SNRs greater than $13 \mathrm{~dB}$. BER of $10^{-3}$ is achieved with $E_{b} / N_{0}$ of $15 \mathrm{~dB}$ in MRC-LDR, which is $18 \mathrm{~dB}$ smaller as compared to $E_{b} / N_{0}$ required for $3 \mathrm{~W}$ Rake, which is $33 \mathrm{~dB}$. This is attributed to the LDR diversity, because, in the LDR three main components are sampled through the use of separate antennas for each direct, lateral, and reflected component, whereas for $3 \mathrm{~W}$-Rake performance suffers because of the bottlenecks in correlation of the three components.

\section{G. LDR Implementation}

Although, the implementation of the LDR is much more complex as compared to conventional matched filter, adaptive switched selection combining is easy to implement, especially with dominant a L-wave or D-wave. AC-LDR can be implemented through zero-forcing (ZF) precoding, which inverts the channel matrix to remove the undesired components. Moreover, optimum MRC combining requires extra hardware due to co-phasing and weighing requirement and is practical through digital signal processing (DSP) hardware. Therefore, optimum MRC combining can be used as benchmark for theoretical performance analysis of the wireless UG channel, as it allows to analyze the performance improvements by using the LDR diversity approach in IOUT. However, keeping in view the importance of high data rate and long distance communications in wireless UG channel, LDR lends itself into consideration for the next generation IOUT system architecture.

\section{Vi. Conclusions}

This paper has reported the performance analysis of different modulation schemes of the UG wireless communication channel in an IOUT system. Adaptive equalization has been shown to be effective against the high delay spread and multipath fading in the UG channel. Novel UG receiver designs for the IOUT have been developed and performance analysis has been done by presenting the BER curves under different soil moisture levels for different depths and distances. Various physical phenomena of soil medium have been shown to impact the BER performance of the UG channel. With change in soil moisture, communications distance, and depth, the IOUT system performance can be determined from our results. The analysis show promising performance improvements with UG 3 W-Rake and LDR receivers in IOUT.

\section{ACKNOWLEDGMENTS}

This work is supported in part by NSF grants NSF CNS1619285, DBI-1331895, and NSF CNS-1423379.

\section{REFERENCES}

[1] T. E. Abrudan, O. Kypris, N. Trigoni, and A. Markham, "Impact of rocks and minerals on underground magneto-inductive communication and localization," IEEE Access, vol. 4, pp. 3999-4010, 2016.

[2] I. F. Akyildiz and E. P. Stuntebeck, "Wireless underground sensor networks: Research challenges," Ad Hoc Networks Journal (Elsevier), vol. 4, pp. 669-686, July 2006.

[3] I. F. Akyildiz, Z. Sun, and M. C. Vuran, "Signal propagation techniques for wireless underground communication networks," Physical Communication Journal (Elsevier), vol. 2, no. 3, pp. 167-183, Sept. 2009.

[4] H. R. Bogena and et.al., "Potential of wireless sensor networks for measuring soil water content variability," Vadose Zone Journal, vol. 9, no. 4, pp. 1002-1013, November 2010.

[5] X. Dong and M. C. Vuran, "Exploiting soil moisture information for adaptive error control in wireless underground sensor networks," in 2013 IEEE Global Communications Conference (GLOBECOM), Dec 2013.

[6] X. Dong and M. C. Vuran, "Impacts of soil moisture on cognitive radio underground networks," in Proc. IEEE BlackSeaCom, Batumi, Georgia, July 2013.

[7] X. Dong and M. C. Vuran, "A channel model for wireless underground sensor networks using lateral waves," in Proc. of IEEE Globecom '11, Houston, TX, December 2011.

[8] X. Dong, M. C. Vuran, and S. Irmak, "Autonomous precision agriculture through integration of wireless underground sensor networks with center pivot irrigation systems," Ad Hoc Networks, vol. 11, no. 7, pp. 1975 1987, 2013.

[9] Ettus Research Website. [Online]. Available: http://www.ettus.com

[10] GNU Radio Website. [Online]. Available: http://www.gnuradio.org

[11] S. Kisseleff, I. Akyildiz, and W. Gerstacker, "Digital signal transmission in magnetic induction based wireless underground sensor networks," IEEE Trans. Communications, vol. 63, no. 6, pp. 2300-2311, June 2015.

[12] J. Proakis and M. Salehi, Digital Communications, 5th ed. McGrawHill, 2007.

[13] A. Salam and M. C. Vuran, "Impacts of soil type and moisture on the capacity of multi-carrier modulation in internet of underground things," in Proc. ICCCN 2016, Hawaii, USA, Aug 2016.

[14] A. Salam and M. C. Vuran, "Smart underground antenna arrays: A soil moisture adaptive beamforming approach," Department of Computer Science and Engineering, University of Nebraska-Lincoln, Tech. Rep. TR-UNL-CSE-2017-0001, January 2017.

[15] A. Salam and M. C. Vuran, "Smart underground antenna arrays: A soil moisture adaptive beamforming approach," in Proc. 36th IEEE INFOCOM 2017, Atlanta, USA, May 2017.

[16] A. Salam, M. C. Vuran, and S. Irmak, "Pulses in the sand: Impulse response analysis of wireless underground channel," in Proc. 35th IEEE INFOCOM 2016, San Francisco, USA, Apr. 2016.

[17] A. Salam, M. C. Vuran, and S. Irmak, "Towards internet of underground things in smart lighting: A statistical model of wireless underground channel," in Proc. 14th IEEE International Conference on Networking, Sensing and Control (IEEE ICNSC), Calabria, Italy, May 2017.

[18] Z. Sun and et.al., "MISE-PIPE: Magnetic induction-based wireless sensor networks for underground pipeline monitoring," Ad Hoc Networks, vol. 9, no. 3, pp. 218-227, 2011.

[19] Z. Sun, P. Wang, M. C. Vuran, M. A. Al-Rodhaan, A. M. Al-Dhelaan, and I. F. Akyildiz, "Border patrol through advanced wireless sensor networks," Ad Hoc Networks, vol. 9, no. 3, pp. 468-477, 2011.

[20] X. Tan, Z. Sun, and I. F. Akyildiz, "Wireless underground sensor networks: Mi-based communication systems for underground applications." IEEE Antennas and Propagation Magazine, vol. 57, no. 4, Aug 2015.

[21] M. J. Tiusanen, "Soil scouts: Description and performance of single hop wireless underground sensor nodes," Ad Hoc Networks, vol. 11, no. 5, pp. $1610-1618,2013$.

[22] M. Vuran, X. Dong, and D. Anthony, "Antenna for wireless underground communication,” Dec. 27 2016, US Patent 9,532,118. [Online]. Available: https://www.google.com/patents/US9532118

[23] M. C. Vuran and I. F. Akyildiz, "Channel model and analysis for wireless underground sensor networks in soil medium," Physical Communication, vol. 3, no. 4, pp. 245-254, December 2010. 